\title{
Transurethral Incision of the Prostate
}

National Cancer Institute

\section{Source}

National Cancer Institute. Transurethral Incision of the Prostate. NCI Thesaurus. Code C113727.

A surgical procedure performed in patients with benign prostatic hyperplasia. An instrument is inserted in the urethra that generates electric current or laser beam, which create incisions in the bladder neck. The incisions result in decreased resistance to the flow of the urine out of the urinary bladder. 